\title{
PINK1 signaling in mitochondrial homeostasis and in aging (Review)
}

\author{
YASUKO KITAGISHI*, NORIKO NAKANO*, MAKO OGINO*, MAYUKO ICHIMURA, \\ AKARI MINAMI and SATORU MATSUDA
}

\begin{abstract}
Department of Food Science and Nutrition, Nara Women's University, Kita-Uoya, Nishimachi, Nara 630-8506, Japan
\end{abstract}
Received June 17, 2016; Accepted December 6, 2016

DOI: $10.3892 / \mathrm{ijmm} .2016 .2827$

\begin{abstract}
Mitochondrial dysfunction is involved in the pathology of Parkinson's disease, an age-associated neurodegenerative disorder. Phosphatase and tensin homolog (PTEN)-induced putative kinase protein 1 (PINK1) is responsible for the most common form of recessive Parkinson's disease. PINK1 is a mitochondrial kinase that is involved in mitrochondrial quality control and promotes cell survival. PINK1 has been shown to protect against neuronal cell death induced by oxidative stress. Accordingly, PINK1 deficiency is associated with mitochondrial dysfunction as well as increased oxidative cellular stress and subsequent neuronal cell death. In addition, several mitochondrial chaperone proteins have been shown to be substrates of the PINK1 kinase. In this review, we discuss recent studies concerning the signaling cascades and molecular mechanisms involved in the process of mitophagy, which is implicated in neurodegeneration and in related aging associated with oxidative stress. Particular attention will be given to the molecular mechanisms proposed to explain the
\end{abstract}

Correspondence to: Professor Satoru Matsuda, Department of Food Science and Nutrition, Nara Women's University, Kita-Uoya, Nishimachi, Nara 630-8506, Japan

E-mail: smatsuda@cc.nara-wu.ac.jp

*Contributed equally

Abbreviations: FoxO3a, forkhead box group O3a; HtrA2, high temperature requirement protein A2; MAPK, mitogen-activated protein kinase; Nrf2, nuclear factor erythroid 2-related factor; PARL, presenilin-associated rhomboid-like serine protease; PARP-1, poly(ADP-ribose) polymerase 1; PINK1, phosphatase and tensin homolog-induced putative kinase protein 1; PGAM5, phosphoglycerate mutase family member 5; PTEN, phosphatase and tensin homolog; ROS, reactive oxygen species; SAPK, stress-activated protein kinase; TRAP1, tumor necrosis factor receptor-associated protein 1 (also known as mitochondrial heat shock protein $75 \mathrm{kDa}$; Hsp75); HSP; Sirt1, sirtuin 1 (silent mating type information regulation 2, S. cerevisiae, homolog 1)

Key words: phosphatase and tensin homolog-induced putative kinase protein 1, Parkin, presenilin-associated rhomboid-like serine protease, high temperature requirement protein A2, mitochondria, oxidative stress, aging, Parkinson's disease effects of natural compounds and/or food ingredients against oxidative stress. Knowledge of the molecular mechanisms involved in this cellular protection could be critical for developing treatments to prevent and control excessive progression of neurodegenerative disorders.

\section{Contents}

1. Introduction

2. Characteristics of the PINK1 protein

3. PINK1 functions in mitochondrial protection

4. PINK1/Parkin/Sirt1/Sirt3/FoxO3 signaling network is related to longevity

5. Diet may contribute to longevity by enhancing the PINK1 signaling network

6. Conclusion

\section{Introduction}

Oxidative stress is thought to play a significant role in the development and progression of certain cell degenerative diseases and aging $(1,2)$. Evidence supports the crucial role of mitochondrial dynamics in the pathogenesis of various diseases. For example, mitochondria are recognized to play an important role in neurodegenerative disorders including multiple sclerosis, Parkinson's disease and Alzheimer's disease, which are characterized by progressive and selective loss of neuronal cell populations (3-5). Aging and these neurodegenerative diseases are associated with a chronic state of oxidative stress and inflammation mediated via organized pathways $(6,7)$. Mitochondria are dynamic organelles and play an essential role in metabolic processes which are prominent targets of oxidative stress. Alterations in mitochondrial activity not only affect the function of individual cells but also metabolism and the lifespan (8). Thus, homeostasis is intensively regulated by a complex interplay between mitochondrial biogenesis and mitochondrial autophagy (mitophagy), an important control mechanism that clears damaged mitochondria, critical for cell survival and for normal cellular functions (9). Mitophagy may also play a key role in mediating apoptosis and in determining their own degradation. Phosphatase and tensin homolog (PTEN)-induced putative kinase protein 1 (PINK1) 

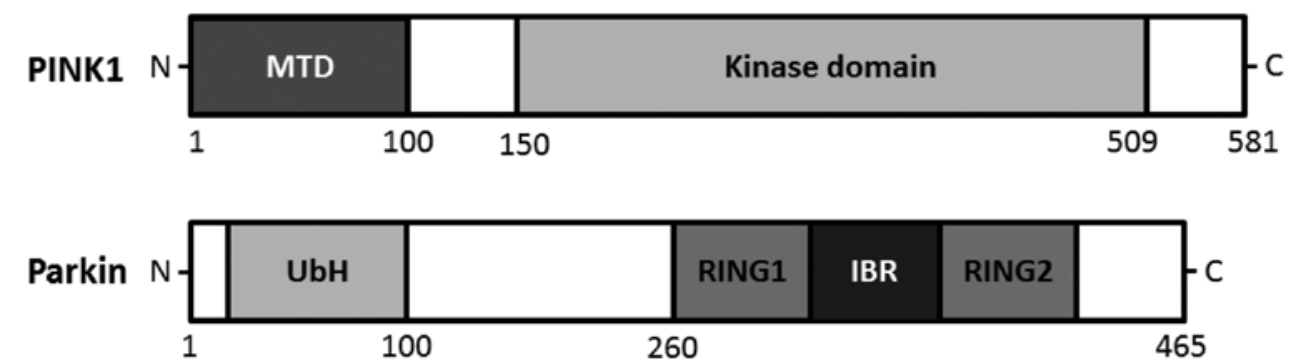

Figure 1. Schematic illustration indicating the domain structures of PINK1 (upper) and Parkin (lower) molecules. Important domain structures for each protein are depicted. PINK1, phosphatase and tensin homolog-induced putative kinase protein 1; MTD, mitochondrial targeting domain; UbH, ubiquitin homology domain; RING1, RING2, RING finger domains; IBR, in-between RING fingers.

is a mitochondrial-targeted serine/threonine kinase, which is linked to autosomal recessive familial Parkinson's disease (10). PINK1 has a protective role against mitochondrial dysfunction and apoptosis with mitochondrial quality control by activating a mitochondrial damage-response signaling pathway (11). It is now clear that mitochondrial oxidant production is controlled by redox signaling under precise physiological conditions. The pathway integrating environmental and genetic stimuli interacts with crucial mitophagy-related effectors to stimulate cellular stress response mechanisms modulating a healthy condition and long lifespan (12). Needless to state, there are many variables involved in how long we live and in maintaining a healthy life. However, it may be possible to help slow the aging process and/or avoid age-related diseases including neurodegenerative disorders, diabetes and heart disease. As aging may be the result of a number of factors, unraveling the regulatory network that governs the crosstalk between mitochondrial biogenesis and mitophagy may enhance our understanding of the molecular mechanisms that regulate mitochondrial function to control aging and age-related diseases. This review provides a concise overview of the cellular functions of mitochondrial kinase PINK1 and the relationship between aging/neurodegeneration and mitochondrial dynamics.

\section{Characteristics of the PINK1 protein}

Localization and stability of PINK1 require catalytic activity of presenilin-associated rhomboid-like serine protease (PARL) that can affect the proteolytic processing of PINK1 (13). In mitochondria, PARL facilitates the cleavage of PINK1, and then mediates differential cleavage of phosphoglycerate mutase family member 5 (PGAM5) depending on the status of the mitochondria (14). In addition, PINK1 processing and localization are indispensable in determining the interaction with E3 ubiquitin ligase Parkin. PINK1 recruits Parkin, a Parkinson's disease-related protein, to mitochondria in order to initiate mitophagy $(15,16)$. Therefore, PARL deficiency also impairs Parkin recruitment to the mitochondria $(15,16)$. Optineurin and NDP52 are the primary receptors for PINK1mediated mitophagy (17). In addition, several molecules acting downstream of PINK1 are activated to maintain mitochondrial homeostasis. The PINK1 gene consists of eight exons, encoding a 581-amino acid protein with a calculated molecular mass of $66 \mathrm{kDa}$ (18). PINK1 mRNA is ubiquitously expressed, and high expression is found in the brain, heart, testis and skeletal muscle (19). Mutations in the PINK1 gene are the most common causes of recessive familial Parkinson's disease (20). An amino terminal targeting signal domain is sufficient for mitochondrial introduction of PINK1, and the carboxyl terminal tail holds regulatory motifs capable of maintaining PINK1 kinase activity with homology to serine/threonine kinases $(21,22)$ (Fig. 1). PINK1 protein can be processed into at least two shorter forms, which are distributed in both the mitochondrial and cytosolic space. Generally, PINK1 is found on the outer and inner mitochondrial membrane. PINK1 phosphorylates a mitochondrial molecular chaperone heat shock protein 75 (Hsp75), also known as mitochondrial heat shock protein $75 \mathrm{kDa}$ [or tumor necrosis factor receptorassociated protein 1 (TRAP1)], which increases neuronal survival withstanding oxidative stress or heat shock by inhibiting the release of cytochrome $c(22,23)$. Serine protease high temperature requirement protein A2 (HtrA2) is released from the inter-membrane space of mitochondria to the cytosol during apoptosis, which may also be regulated by PINK1 (24). However, whether HtrA2 is a direct PINK1 substrate is fairly uncertain. Targeted deletion of HtrA 2 affects mitochondrial dysfunction leading to a neurodegenerative disorder with Parkinsonian phenotype in experimental mice (25). In addition, PINK1 may also interact with Beclin1, a crucial autophagic protein implicated in the pathogenesis of Alzheimer's disease and/or Huntington's disease (26). Interaction of PINK1 with Beclin1 was found to augment autophagy (27). Consequently, physiological PINK1 substrates may be localized in the outer membrane of mitochondria or possibly in the cytosol adjacent to the mitochondrial surface. Cytoplasmic PINK1 is degraded by proteasomes $(28,29)$. It is feasible that differences in cell viability initiated from PINK1 inactivation may affect several substrates through other kinases such as p38 stressactivated protein kinase (SAPK)/mitogen-activated protein kinase (MAPK) $(28,29)$.

\section{PINK1 functions in mitochondrial protection}

The importance of PINK1 in the mitochondria is reflected by cell-protective properties for counteracting oxidative stress. When mitochondria are compromised by cellular depolarization, PINK1 accumulates on the mitochondrial surface where it recruits the Parkin protein from the cytosol, which in turn mediates the degradation of mitochondria termed mitophagy (Fig. 2) (30,31). Knockdown of endogenous PINK1 and/or loss of PINK1 leads to alterations in mitochondrial homeostasis as evidenced by increased mitochondrial 


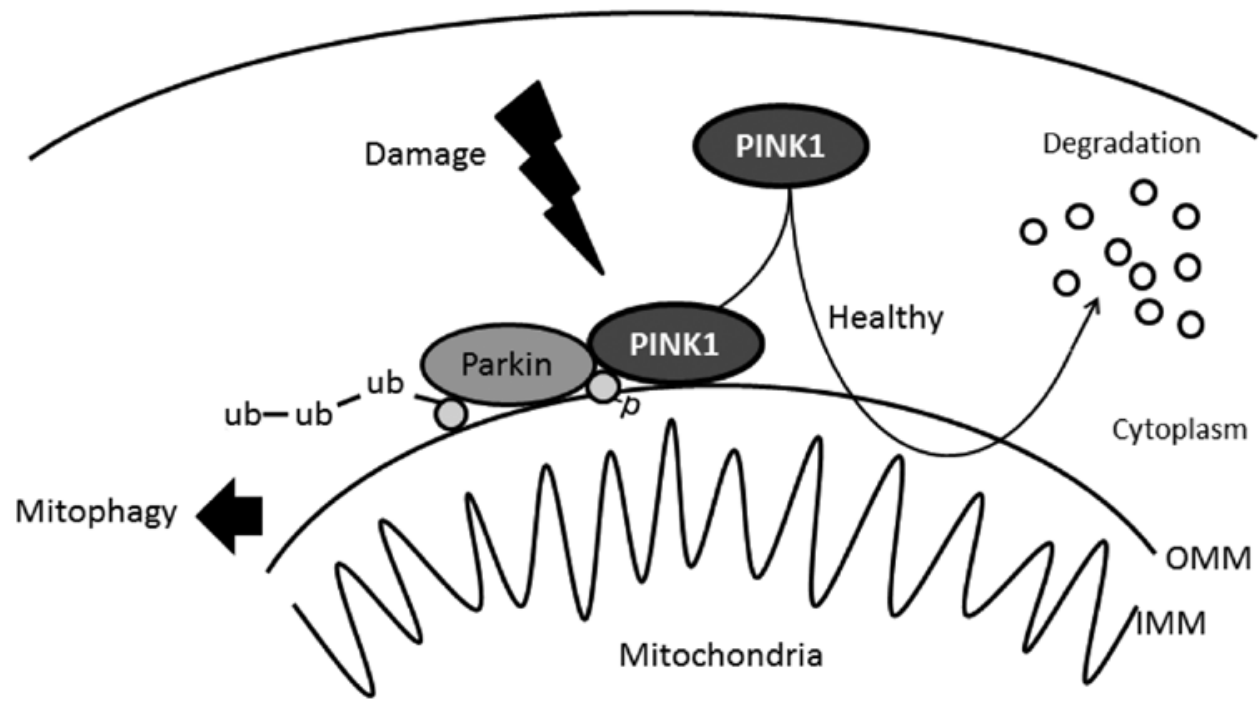

Figure 2. Hypothetical schematic image of the PINK1 regulatory pathway with Parkin-mediated mitophagy. In healthy cells under a steady state, PINK1 is degraded within mitochondria. This may be halted by mitochondrial damage resulting in PINK1 and Parkin accumulation in the outer membrane of mitochondria. Parkin is assumed to be ubiquitinated (ub) by an unidentified substrate (grey circle) resulting in the induction of mitophagy. In addition, PINK1 phosphorylates ubiquitin to activate Parkin ubiquitin ligase activity. Note that some critical pathways have been omitted for clarity. PINK1, phosphatase and tensin homolog-induced putative kinase protein 1; OMM, outer mitochondrial membrane; IMM, inner mitochondrial membrane.

reactive oxygen species (ROS) carrying an escalation in mitophagy (32). Protective activity of PINK1 in maintaining mitochondrial health depends on its mitochondrial localization $(33,34)$. Stable silencing of PINK1 may have an incidental role in the activation of mitophagy $(33,34)$. Thus, PINK1 plays a pivotal role in mitochondrial quality control via the regulation of mitophagy and/or mitochondrial maintenance. However, excessive rates of mitophagy have been found to be harmful $(35,36)$. In healthy mitochondria, PINK1 is promptly degraded in a process comprising mitochondrial proteases and proteasomes $(37,38)$. As mentioned above, mitochondrial protease PARL may mediate differential cleavage of PINK1 depending on the health status of mitochondria (14). Stability of PINK1 requires catalytic activity of PARL $(14,15)$. PARL deficiency impairs Parkin recruitment to mitochondria (15), suggesting that PINK1 localization is important in defining the interaction with Parkin. Complete PINK1 is free to be released into the mitochondrial inter-membrane space or the cytosol. Blockage of PARL processing and the import of PINK1 upon depolarization of the mitochondrial membrane, leads to accumulation of PINK1 precursor (39). Targeting of this precursor protein to the outer mitochondrial membrane has been shown to initiate mitophagy $(22,40)$. Hence, the removal of PINK1 may act as a cellular checkpoint for the control of mitochondrial reliability, indicating that interactions of PINK1 with Parkin promote healthy mitochondrial homeostasis. Expression of full-length PINK1 is essential for mitochondrial Parkin recruitment. Transient expression of Parkin further augments mitochondrial mitophagy, resulting in cytoprotection of mitochondrial networks (41). Following severe mitochondrial damage, however, PINK1 facilitates aggregation of depolarized mitochondria through interactions with Parkin (27). Under conditions of PINK1 deficiency, mitochondrial quality control is eventually compromised. Parkin can be phosphorylated by PINK1 in its RING finger domain, which may promote translocation of Parkin to mitochondria
(42). It has been reported to facilitate the clearance of depolarized mitochondria via mitophagy $(43,44)$. Failure of this quality control ultimately results in cell death. Therefore, PINK1 and Parkin synergistically participate in a common mitochondrial complex signaling pathway. Recently, it has been reported that PINK1 phosphorylates ubiquitin to activate Parkin E3 ubiquitin ligase activity (45) (Fig. 2). This phosphorylation may be required for the activation of Parkin for full activation to induce selective autophagy of damaged mitochondria. Phosphorylation of Parkin helps to prepare the ubiquitin ligase enzyme for activation by ubiquitin phospho-Ser65 (46). The phosphorylation-dependent interaction between ubiquitin and Parkin suggests that phosphorylated ubiquitin unravels autoinhibition of this signaling (47). These findings demonstrate that phosphorylated ubiquitin is a Parkin activator (47).

\section{PINK1/Parkin/Sirt1/Sirt3/FoxO3 signaling network is related to longevity}

Dysfunction of mitochondria is a common feature in aging and in neurodegeneration (48). In some cases, mitochondrial abnormalities appear to be caused by decreased activation of Sirt1 initiated by hyperactivation of the DNA damage sensor PARP-1 [poly(ADP-ribose) polymerase 1] leading to mitochondrial membrane depolarization, PINK1 cleavage, and impaired mitophagy (49). Notably, DNA repair is an essential mechanism for cell survival, and various defects in the DNA repair system lead to accelerated aging (49). Sirtuin 1 (silent mating type information regulation $2, S$. cerevisiae, homolog 1) (Sirt1) is the most prominent member of the mammalian class III histone deacetylase family implicated in healthy lifespan and longevity (50). A nuclear-mitochondrial crosstalk seems to be critical for the maintenance of mitochondrial health (48), in which the function of Sirt1 appears to be important $(48,51)$. Sirt1 regulates the DNA repair and the related metabolism by deacetylating target proteins such 


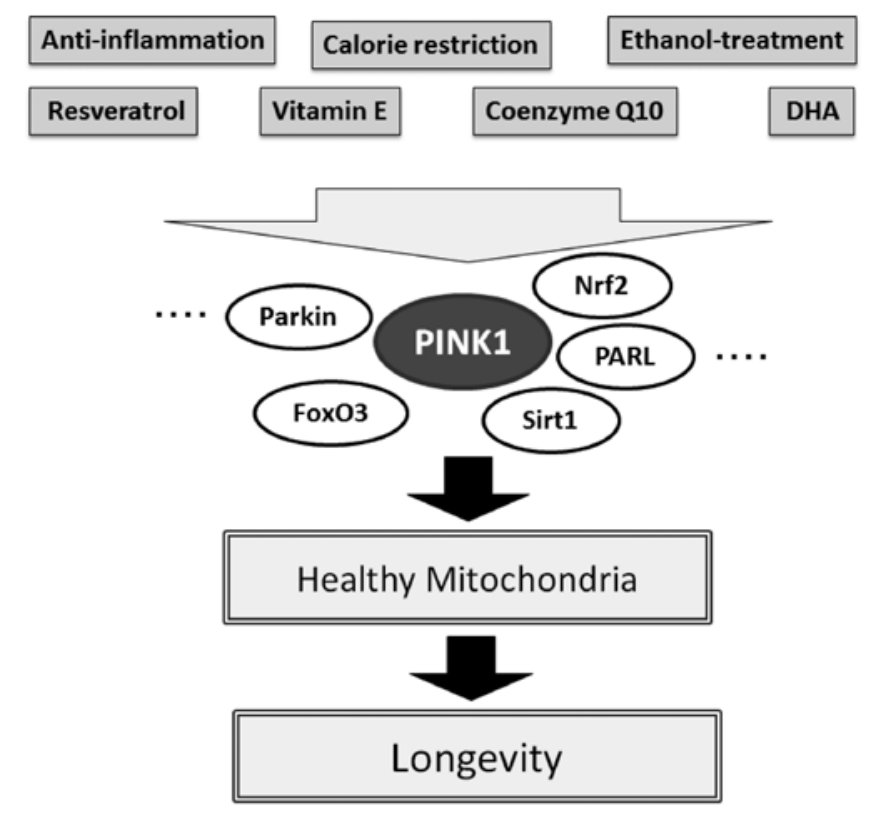

Figure 3. Implication of mitochondrial health induced by the PINK1, Parkin, Sirt1, Nrf2, FoxO3 and PARL network. Various food ingredients and specific diets may contribute to mitochondrial health via the PINK1 network, eventually leading to longevity. Note that some critical molecules have been omitted for clarity. PINK1, phosphatase and tensin homolog-induced kinase 1; Nrf2, nuclear factor erythroid 2-related factor; FoxO3, forkhead box group O3a; PARL, presenilin-associated rhomboid-like serine protease.

as p53 tumor suppressor. The mitochondrial abnormalities may be caused by decreased activation of SIRT1 triggered by DNA damage. As PINK1 activates Parkin which translocates to depolarized mitochondria, the PINK1/Parkin/Sirt1 pathway may also act synergistically to promote mitochondrial degradation by mitophagy in order to protect cells (52). In addition, several members of transcription factors control PINK1 transcription. For example, it is known that mitochondrial Sirt3 interacts and regulates the activity of FoxO3a, the Forkhead box subgroup $\mathrm{O}$ (FoxO), acting through the conserved FoxO binding elements in mitochondria. Overexpression of the Sirt3 gene increases FoxO3a DNA binding activity as well as FoxO3a-dependent gene expression including PINK1 (53). Induction of PINK1 by FoxO3a is crucial for critical survival signals in normal cells, as depletion of PINK1 sensitizes those cells to cell death (53). Furthermore, increased FoxO3a expression reduces both ROS and thereby apoptotic cell death (54). Multi-functional heat shock protein $40 \mathrm{kDa}$ (HSP40) was found to decrease PINK1 mRNA level by binding to FoxO3a that interacts with the PINK1 promoter encouraging its transcriptional activity (55). FoxO3a alteration is determinedly linked to the progression of various types of cancer, fibrosis, and other types of disease. Recently, several studies have revealed the importance of Sirt3 along with FoxO3a in addition to Sirt1 with resveratrol in preventing premature aging $(56,57)$. The favorable protective effect of resveratrol has been shown to be diminished upon pharmacological inhibition of Sirtl by using sirtinol (56). Sirt1 in cooperation with Sirt3 activates FoxO3a thereby promoting the stimulation of the PINK-1/Parkin pathway leading to mitophagy. The importance of mitophagy has been revealed as it encourages a healthy pool of mitochon- dria and prevents premature aging (49). In addition, the protein level of nuclear factor erythroid 2-related factor (Nrf2) is also involved in stress resistance and longevity $(58,59)$. Notably, PINK1 expression is positively regulated by NRF2 activity and the Nrf2/PINK1 signaling axis is deeply involved in cell survival and longevity (60).

\section{Diet may contribute to longevity by enhancing the PINK1 signaling network}

Various anti-inflammatory drugs could be used as a supplement to scavenge ROS and hence improve the survival span of several types of cells (61). Antioxidants also act as inhibitors of radical production processes by removing harmful ROS formed during normal cellular metabolism $(62,63)$. It is known that the polyphenolic natural antioxidant resveratrol, present in red wine and grapes, exhibits a number of pharmacological effects including anti-inflammation, antioxidation, anti-apoptosis, subsequently promoting longevity $(64,65)$. Resveratrol can pass through the blood-brain barrier and is water soluble (66). The anti-aging effects of resveratrol are believed to be mediated by activation of Sirt1 and the consequent reduced oxidative stress. As mentioned above, several studies have revealed that Sirt3 along with FoxO3 in addition to Sirtl are of importance in promoting the anti-aging function of resveratrol, which promotes the initial mitochondrial signaling response to activate PINK1 thereby promoting mitophagy. It is appealing to speculate that resveratrol promotes anti-aging through this PINK1/Sirt1/FoxO3a signaling pathway comprising mitophagy (Fig. 3). For example, PINK1 is overexpressed in mitochondria of hepatocytes of ethanol-treated rats, in which treatment with a small amount of ethanol represent a possible protective mechanism via the stimulation of mitophagy (67). Dysfunctional mitophagy in response to gluco-lipotoxicities may play an important role in several liver diseases including hepatosteatosis (68). In addition, metformin is known to improve hepatosteatosis by inducing Sirt1-mediated mitophagy (68). Dysregulation of the Parkin pathway by metabolic malregulation may contribute to the pathogenesis of Parkinson's disease and metformin may exert a neuroprotective effect on neuronal disease via the restoration of Parkin (69). Furthermore, DHA attenuates insulin resistance in obese mice through the activation of Sirt1 (70). High fat dietfed animals were found to have reduced mRNA expression of PARL (71). Antioxidant vitamins and vitamin-like substances, such as vitamin E and coenzyme Q10, have been used in the treatment of neurodegenerative diseases with noteworthy efficacy (72). It is well known that a high intake of fruits and vegetables rich in antioxidant vitamins is inversely related to the incidence of neurodegenerative diseases. In contrast, treatment with an isoflavonoid pesticide, which inhibits mitochondrial complex I activity creating an environment of oxidative stress in cells, can produce nigrastriatal neuronal cell-loss and it produces an experimental animal model of Parkinson's disease with similar symptoms $(73,74)$. The level of mitochondrial PINK1 protein has been shown to be increased after pesticide exposure $(73,74)$. Cell protective proteins including Parkin and heat shock proteins (HSPs) may play fundamental roles even in slowing disease progression (73,74). Expression of Parkin and PINK1 was found to be significantly attenuated in a rat group fed a low-protein diet supplemented with various types of 
keto-acids (75). Therefore, oxidative damage can be reduced by adhering to certain diets with specific vitamins and/or restriction of calorie intake controlling hyperglycemia. Consequently, nutritional control related to metabolic antioxidation may encourage longevity followed by healthy mitochondria with minimal degeneration of cells.

\section{Conclusion}

Mitochondria are involved in cell stress-induced programmed cell death, which also contributes to the regulation of mitochondrial dynamics and mitophagy. In terms of the effect of specific ROS on mitophagy, future research is needed to ascertain how to maintain mitochondrial quality and ensure cellular homeostasis. PINK1 protein may play key roles in the primary line of mitochondrial quality control and in monitoring respiratory function under conditions of oxidative stress. However, low levels of redox signaling may be essential for normal mitophagy. Although the detailed physiological substrate of PINK1 is not fully determined, it is clear that kinase activity is important in many aspects of mitochondrial function in addition to mitophagy (76). Hence, reduction in PINK1 activity may have eventual lethal consequences on mitochondria and/ or cells. Enhancing pathways that promote dynamic mitophagy may delay age-related diseases by promoting a healthy pool of viable mitochondria in cells and by sustaining good energy metabolism. Future experimental research may further clarify the mitochondrial protective roles of PINK1 and Parkin.

\section{Acknowledgements}

This study was supported by grants from JSPS KAKENHI (nos. 26-12035 and 24240098).

\section{References}

1. Prasad AS: Zinc: an antioxidant and anti-inflammatory agent: role of zinc in degenerative disorders of aging. J Trace Elem Med Biol 28: 364-371, 2014.

2. Tebay LE, Robertson H, Durant ST, Vitale SR, Penning TM, Dinkova-Kostova AT and Hayes JD: Mechanisms of activation of the transcription factor Nrf2 by redox stressors, nutrient cues, and energy status and the pathways through which it attenuates degenerative disease. Free Radic Biol Med 88: 108-146, 2015.

3. Campbell GR, Worrall JT and Mahad DJ: The central role of mitochondria in axonal degeneration in multiple sclerosis. Mult Scler 20: 1806-1813, 2014.

4. Erpapazoglou Z and Corti O: The endoplasmic reticulum/mitochondria interface: A subcellular platform for the orchestration of the functions of the PINK1-Parkin pathway? Biochem Soc Trans 43: 297-301, 2015.

5. Kaminsky YG, Tikhonova LA and Kosenko EA: Critical analysis of Alzheimer's amyloid-beta toxicity to mitochondria. Front Biosci (Landmark Ed) 20: 173-197, 2015.

6. Oyewole AO and Birch-Machin MA: Mitochondria-targeted antioxidants. FASEB J 29: 4766-4771, 2015.

7. Tocchi A, Quarles EK, Basisty N, Gitari L and Rabinovitch PS Mitochondrial dysfunction in cardiac aging. Biochim Biophys Acta 1847: 1424-1433, 2015.

8. Pan Y, Nishida Y, Wang M and Verdin E: Metabolic regulation, mitochondria and the life-prolonging effect of rapamycin: A mini-review. Gerontology 58: 524-530, 2012.

9. Quirós PM, Langer T and López-Otín C: New roles for mitochondrial proteases in health, ageing and disease. Nat Rev Mol Cell Biol 16: 345-359, 2015.

10. Durcan TM and Fon EA: The three 'P's of mitophagy: PARKIN, PINK1, and post-translational modifications. Genes Dev 29: 989-999, 2015
11. Wilhelmus MM, van der Pol SM, Jansen Q, Witte ME, van der Valk P, Rozemuller AJ, Drukarch B, de Vries HE and Van Horssen J: Association of Parkinson disease-related protein PINK1 with Alzheimer disease and multiple sclerosis brain lesions. Free Radic Biol Med 50: 469-476, 2011.

12. Russell AP, Foletta VC, Snow RJ and Wadley GD: Skeletal muscle mitochondria: A major player in exercise, health and disease. Biochim Biophys Acta 1840: 1276-1284, 2014.

13. Jin SM, Lazarou M, Wang C, Kane LA, Narendra DP and Youle RJ: Mitochondrial membrane potential regulates PINK1 import and proteolytic destabilization by PARL. J Cell Biol 191: 933-942, 2010.

14. Sekine S, Kanamaru Y, Koike M, Nishihara A, Okada M, Kinoshita H, Kamiyama M, Maruyama J, Uchiyama Y, Ishihara N, et al: Rhomboid protease PARL mediates the mitochondrial membrane potential loss-induced cleavage of PGAM5. J Biol Chem 287: 34635-34645, 2012.

15. Shi G, Lee JR, Grimes DA, Racacho L, Ye D, Yang H, Ross OA, Farrer M, McQuibban GA and Bulman DE: Functional alteration of PARL contributes to mitochondrial dysregulation in Parkinson's disease. Hum Mol Genet 20: 1966-1974, 2011.

16. Pickrell AM and Youle RJ: The roles of PINK1, parkin, and mitochondrial fidelity in Parkinson's disease. Neuron 85: 257-273, 2015.

17. Lazarou M, Sliter DA, Kane LA, Sarraf SA, Wang C, Burman JL, Sideris DP, Fogel AI and Youle RJ: The ubiquitin kinase PINK1 recruits autophagy receptors to induce mitophagy. Nature 524: 309-314, 2015.

18. Weihofen A, Ostaszewski B, Minami Y and Selkoe DJ: Pink1 Parkinson mutations, the Cdc37/Hsp90 chaperones and Parkin all influence the maturation or subcellular distribution of Pink1. Hum Mol Genet 17: 602-616, 2008.

19. d'Amora M, Angelini C, Marcoli M, Cervetto C, Kitada T and Vallarino M: Expression of PINK1 in the brain, eye and ear of mouse during embryonic development. J Chem Neuroanat 41: 73-85, 2011.

20. Narendra D, Walker JE and Youle R: Mitochondrial quality control mediated by PINK1 and Parkin: Links to parkinsonism. Cold Spring Harb Perspect Biol 4: a011338, 2012.

21. Sim CH, Lio DS, Mok SS, Masters CL, Hill AF, Culvenor JG and Cheng HC: C-terminal truncation and Parkinson's disease-associated mutations down-regulate the protein serine/threonine kinase activity of PTEN-induced kinase-1. Hum Mol Genet 15: 3251-3262, 2006

22. Okatsu K, Oka T, Iguchi M, Imamura K, Kosako H, Tani N, Kimura M, Go E, Koyano F, Funayama M, et al: PINK1 autophosphorylation upon membrane potential dissipation is essential for Parkin recruitment to damaged mitochondria. Nat Commun 3: 1016, 2012

23. Pridgeon JW, Olzmann JA, Chin LS and Li L: PINK1 protects against oxidative stress by phosphorylating mitochondrial chaperone TRAP1. PLoS Biol 5: e172, 2007.

24. Gaki GS and Papavassiliou AG: Oxidative stress-induced signaling pathways implicated in the pathogenesis of Parkinson's disease. Neuromolecular Med 16: 217-230, 2014.

25. Plun-Favreau H, Klupsch K, Moisoi N, Gandhi S, Kjaer S, Frith D, Harvey K, Deas E, Harvey RJ, McDonald N, et al: The mitochondrial protease HtrA2 is regulated by Parkinson's diseaseassociated kinase PINK1. Nat Cell Biol 9: 1243-1252, 2007.

26. Lenzi P, Marongiu R, Falleni A, Gelmetti V, Busceti CL, Michiorri S, Valente EM and Fornai F: A subcellular analysis of genetic modulation of PINK1 on mitochondrial alterations, autophagy and cell death. Arch Ital Biol 150: 194-217, 2012.

27. Chu CT: A pivotal role for PINK1 and autophagy in mitochondrial quality control: Implications for Parkinson disease. Hum Mol Genet 19: R28-R37, 2010.

28. Choi I, Kim J, Jeong HK, Kim B, Jou I, Park SM, Chen L, Kang UJ, Zhuang X and Joe EH: PINK1 deficiency attenuates astrocyte proliferation through mitochondrial dysfunction, reduced AKT and increased p38 MAPK activation, and downregulation of EGFR. Glia 61: 800-812, 2013.

29. Lin W and Kang UJ: Characterization of PINK1 processing, stability, and subcellular localization. J Neurochem 106: 464-474, 2008.

30. Wei H, Liu L and Chen Q: Selective removal of mitochondria via mitophagy: Distinct pathways for different mitochondrial stresses. Biochim Biophys Acta 1853: 2784-2790, 2015.

31. Eiyama A and Okamoto K: PINK1/Parkin-mediated mitophagy in mammalian cells. Curr Opin Cell Biol 33: 95-101, 2015.

32. Dias V, Junn E and Mouradian MM: The role of oxidative stress in Parkinson's disease. J Parkinsons Dis 3: 461-491, 2013. 
33. McCoy MK, Kaganovich A, Rudenko IN, Ding J and Cookson MR: Hexokinase activity is required for recruitment of parkin to depolarized mitochondria. Hum Mol Genet 23: 145-156, 2014.

34. Michiorri S, Gelmetti V, Giarda E, Lombardi F, Romano F Marongiu R, Nerini-Molteni S, Sale P, Vago R, Arena G, et al The Parkinson-associated protein PINK1 interacts with Beclin1 and promotes autophagy. Cell Death Differ 17: 962-974, 2010.

35. Cherra SJ III and Chu CT: Autophagy in neuroprotection and neurodegeneration: A question of balance. Future Neurol 3 : 309-323, 2008.

36. Cherra SJ III, Dagda RK and Chu CT: Review: autophagy and neurodegeneration: survival at a cost? Neuropathol App Neurobiol 36: 125-132, 2010.

37. Moriwaki Y, Kim YJ, Ido Y, Misawa H, Kawashima K, Endo S and Takahashi R: L347P PINK1 mutant that fails to bind to Hsp90/Cdc37 chaperones is rapidly degraded in a proteasomedependent manner. Neurosci Res 61: 43-48, 2008.

38. Sun F, Kanthasamy A, Anantharam V and Kanthasamy AG: Environmental neurotoxic chemical-induced ubiquitin proteasome system dysfunction in the pathogenesis and progression of Parkinson's disease. Pharmacol Ther 114: 327-344 2007.

39. Meissner C, Lorenz H, Weihofen A, Selkoe DJ and Lemberg MK The mitochondrial intramembrane protease PARL cleaves human Pink1 to regulate Pink1 trafficking. J Neurochem 117: 856-867, 2011.

40. Matsuda S, Kitagishi Y and Kobayashi M: Function and characteristics of PINK1 in mitochondria. Oxid Med Cell Longev 2013: 601587, 2013.

41. Dagda RK and Chu CT: Mitochondrial quality control: Insights on how Parkinson's disease related genes PINK1, parkin, and Omi/HtrA2 interact to maintain mitochondrial homeostasis. J Bioenerg Biomembr 41: 473-479, 2009.

42. Kim Y, Park J, Kim S, Song S, Kwon SK, Lee SH, Kitada T, Kim JM and Chung J: PINK1 controls mitochondrial localization of Parkin through direct phosphorylation. Biochem Biophys Res Commun 377: 975-980, 2008.

43. Narendra D, Tanaka A, Suen DF and Youle RJ: Parkin is recruited selectively to impaired mitochondria and promotes their autophagy. J Cell Biol 183: 795-803, 2008.

44. Narendra DP and Youle RJ: Targeting mitochondrial dysfunction: Role for PINK1 and Parkin in mitochondrial quality control. Antioxid Redox Signal 14: 1929-1938, 2011.

45. Kane LA, Lazarou M, Fogel AI, Li Y, Yamano K, Sarraf SA, Banerjee $S$ and Youle RJ: PINK1 phosphorylates ubiquitin to activate Parkin E3 ubiquitin ligase activity. J Cell Biol 205: 143-153, 2014

46. Kazlauskaite A, Kondapalli C, Gourlay R, Campbell DG Ritorto MS, Hofmann K, Alessi DR, Knebel A, Trost M and Muqit MM: Parkin is activated by PINK1-dependent phosphorylation of ubiquitin at Ser65. Biochem J 460: 127-139, 2014.

47. Koyano F, Okatsu K, Kosako H, Tamura Y, Go E, Kimura M, Kimura Y, Tsuchiya H, Yoshihara H, Hirokawa T, et al: Ubiquitin is phosphorylated by PINK1 to activate parkin. Nature 510 : $162-166,2014$

48. Fang EF, Scheibye-Knudsen M, Brace LE, Kassahun H, SenGupta T, Nilsen H, Mitchell JR, Croteau DL and Bohr VA: Defective mitophagy in XPA via PARP-1 hyperactivation and NAD(+)/SIRT1 reduction. Cell 157: 882-896, 2014.

49. Scheibye-Knudsen M, Fang EF, Croteau DL and Bohr VA: Contribution of defective mitophagy to the neurodegeneration in DNA repair-deficient disorders. Autophagy 10: 1468-1469, 2014.

50. Tang BL: Sirt1 and the mitochondria. Mol Cells 39: 87-95, 2016.

51. Greene AW, Grenier K, Aguileta MA, Muise S, Farazifard R Haque ME, McBride HM, Park DS and Fon EA: Mitochondrial processing peptidase regulates PINK1 processing, import and Parkin recruitment. EMBO Rep 13: 378-385, 2012.

52. Das S, Mitrovsky G, Vasanthi HR and Das DK: Antiaging properties of a grape-derived antioxidant are regulated by mitochondrial balance of fusion and fission leading to mitophagy triggered by a signaling network of Sirt1-Sirt3-Foxo3-PINK1PARKIN. Oxid Med Cell Longev 2014: 345105, 2014.

53. Mei Y, Zhang Y, Yamamoto K, Xie W, Mak TW and You H: FOXO3a-dependent regulation of Pink1 (Park6) mediates survival signaling in response to cytokine deprivation. Proc Natl Acad Sci USA 106: 5153-5158, 2009.

54. Sengupta A, Molkentin JD, Paik JH, DePinho RA and Yutzey KE: FoxO transcription factors promote cardiomyocyte survival upon induction of oxidative stress. J Biol Chem 286: 7468-7478, 2011.
55. Requejo-Aguilar R, Lopez-Fabuel I, Jimenez-Blasco D, Fernandez E, Almeida A and Bolaños JP: DJ1 represses glycolysis and cell proliferation by transcriptionally upregulating Pink1. Biochem J 467: 303-310, 2015.

56. Sin TK, Yung BY, Yip SP, Chan LW, Wong CS, Tam EW and Siu PM: SIRT1-dependent myoprotective effects of resveratrol on muscle injury induced by compression. Front Physiol 6: 293, 2015.

57. Lin CH, Lin CC, Ting WJ, Pai PY, Kuo CH, Ho TJ, Kuo WW, Chang $\mathrm{CH}$, Huang $\mathrm{CY}$ and Lin WT: Resveratrol enhanced FOXO3 phosphorylation via synergetic activation of SIRT1 and PI3K/Akt signaling to improve the effects of exercise in elderly rat hearts. Age (Dordr) 36: 9705, 2014.

58. Castillo-Quan JI, Li L, Kinghorn KJ, Ivanov DK, Tain LS, Slack C, Kerr F, Nespital T, Thornton J, Hardy J, et al: Lithium promotes longevity through GSK3/NRF2-dependent hormesis. Cell Rep 15: 638-650, 2016.

59. Lewis KN, Wason E, Edrey YH, Kristan DM, Nevo E and Buffenstein R: Regulation of Nrf2 signaling and longevity in naturally long-lived rodents. Proc Natl Acad Sci USA 112: 3722-3727, 2015.

60. Murata H, Takamatsu H, Liu S, Kataoka K, Huh NH and Sakaguchi M: NRF2 regulates PINK1 expression under oxidative stress conditions. PLoS One 10: e0142438, 2015.

61. Huang ST, Ho CS, Lin CM, Fang HW and Peng YX: Development and biological evaluation of $\mathrm{C}(60)$ fulleropyrrolidine-thalidomide dyad as a new anti-inflammation agent. Bioorg Med Chem 16: 8619-8626, 2008

62. Amorati R, Valgimigli L, Panzella L, Napolitano A and d'Ischia M: 5-S-lipoylhydroxytyrosol, a multidefense antioxidant featuring a solvent-tunable peroxyl radical-scavenging 3-thio1,2-dihydroxybenzene motif. J Org Chem 78: 9857-9864, 2013.

63. Kenneth NS, Hucks GE Jr, Kocab AJ, McCollom AL and Duckett CS: Copper is a potent inhibitor of both the canonical and non-canonical NFkB pathways. Cell Cycle 13: 1006-1014, 2014.

64. Deng ZY, Hu MM, Xin YF and Gang C: Resveratrol alleviates vascular inflammatory injury by inhibiting inflammasome activation in rats with hypercholesterolemia and vitamin D2 treatment. Inflamm Res 64: 321-332, 2015.

65. Baolin L, Inami Y, Tanaka H, Inagaki N, Inuma $\mathrm{M}$ and Nagai $\mathrm{H}$ : Resveratrol inhibits the release of mediators from bone marrowderived mouse mast cells in vitro. Planta Med 70: 305-309, 2004

66. Wang Q, Xu J, Rottinghaus GE, Simonyi A, Lubahn D, Sun GY and Sun AY: Resveratrol protects against global cerebral ischemic injury in gerbils. Brain Res 958: 439-447, 2002.

67. Eid N, Ito Y, Maemura K and Otsuki Y: Elevated autophagic sequestration of mitochondria and lipid droplets in steatotic hepatocytes of chronic ethanol-treated rats: An immunohistochemical and electron microscopic study. J Mol Histol 44: 311-326, 2013.

68. Song YM, Lee WK, Lee YH, Kang ES, Cha BS and Lee BW: Metformin restores Parkin-mediated mitophagy, suppressed by cytosolic p53. Int J Mol Sci 17: E122, 2016.

69. Khang R, Park C and Shin JH: Dysregulation of parkin in the substantia nigra of $\mathrm{db} / \mathrm{db}$ and high-fat diet mice. Neuroscience 294 : 182-192, 2015.

70. Luo X, Jia R, Yao Q, Xu Y, Luo Z, Luo X and Wang N: Docosahexaenoic acid attenuates adipose tissue angiogenesis and insulin resistance in high fat diet-fed middle-aged mice via a sirt1dependent mechanism. Mol Nutr Food Res 60: 871-885, 2016.

71. Borengasser SJ, Faske J, Kang P, Blackburn ML, Badger TM and Shankar K: In utero exposure to prepregnancy maternal obesity and postweaning high-fat diet impair regulators of mitochondrial dynamics in rat placenta and offspring. Physiol Genomics 46: 841-850, 2014.

72. Ono K and Yamada M: Vitamin A potently destabilizes preformed alpha-synuclein fibrils in vitro: Implications for Lewy body diseases. Neurobiol Dis 25: 446-454, 2007.

73. Casarejos MJ, Menéndez J, Solano RM, Rodríguez-Navarro JA, García de Yébenes J and Mena MA: Susceptibility to rotenone is increased in neurons from parkin null mice and is reduced by minocycline. J Neurochem 97: 934-946, 2006.

74. Sonia Angeline M, Chaterjee P, Anand K, Ambasta RK and Kumar P: Rotenone-induced parkinsonism elicits behavioral impairments and differential expression of parkin, heat shock proteins and caspases in the rat. Neuroscience 220: 291-301, 2012

75. Zhang YY, Huang J, Yang M, Gu LJ, Ji JY, Wang LJ and Yuan WJ: Effect of a low-protein diet supplemented with ketoacids on autophagy and inflammation in 5/6 nephrectomized rats. Biosci Rep 35: e00263, 2015.

76. Koh $\mathrm{H}$ and Chung J: PINK1 as a molecular checkpoint in the maintenance of mitochondrial function and integrity. Mol Cells 34: 7-13, 2012. 